\title{
ERRATUM
}

Open Access

\section{Erratum to: Cancer progression by breast tumors with Pit-1-overexpression is blocked by inhibition of metalloproteinase (MMP)-13}

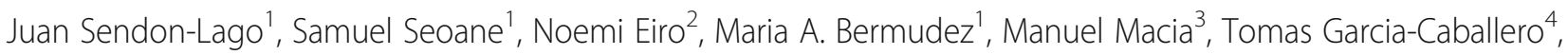
Francisco J. Vizoso ${ }^{2}$ and Roman Perez-Fernandez ${ }^{1 *}$

\section{Erratum}

After the publication of this work [1] an error was noticed in Fig. 4d and 4f. In the migration and invasion assays the same image was used accidentally for the Pit- $1+$ shMMP1 and Pit- $1+$ shMMP-13. The corrected figure is shown below. The error does not affect the findings or conclusion of the article. We apologize for this error.

\begin{abstract}
Author details
${ }^{1}$ Department of Physiology- Center for Research in Molecular Medicine and Chronic Diseases (CIMUS), School of Medicine, University of Santiago de Compostela, 15782 Santiago de Compostela, Spain. ${ }^{2}$ Unidad de Investigación, Fundacion Hospital de Jove, 33290 Gijón, Spain. ${ }^{3}$ Departments of Obstetrics and Gynecology, School of Medicine, University of Santiago de Compostela, 15782 Santiago de Compostela, Spain. ${ }^{4}$ Departments of Morphological Sciences, School of Medicine, University of Santiago de Compostela, 15782 Santiago de Compostela, Spain.
\end{abstract}

Received: 6 March 2017 Accepted: 6 March 2017 Published online: 28 March 2017

\section{References}

1. Sendon-Lago J, Seoane S, Eiro N, Bermudez MA, Macia M, Garcia-Caballero T, Vizoso FJ, Perez-Fernandez R. Cancer progression by breast tumors with Pit-1-overexpression is blocked by inhibition of Metalloproteinase (MMP)-13. Breast Cancer Res. 2014;16:505.

\footnotetext{
* Correspondence: roman.perez.fernandez@usc.es

1Department of Physiology- Center for Research in Molecular Medicine and Chronic Diseases (CIMUS), School of Medicine, University of Santiago de Compostela, 15782 Santiago de Compostela, Spain

Full list of author information is available at the end of the article
} 

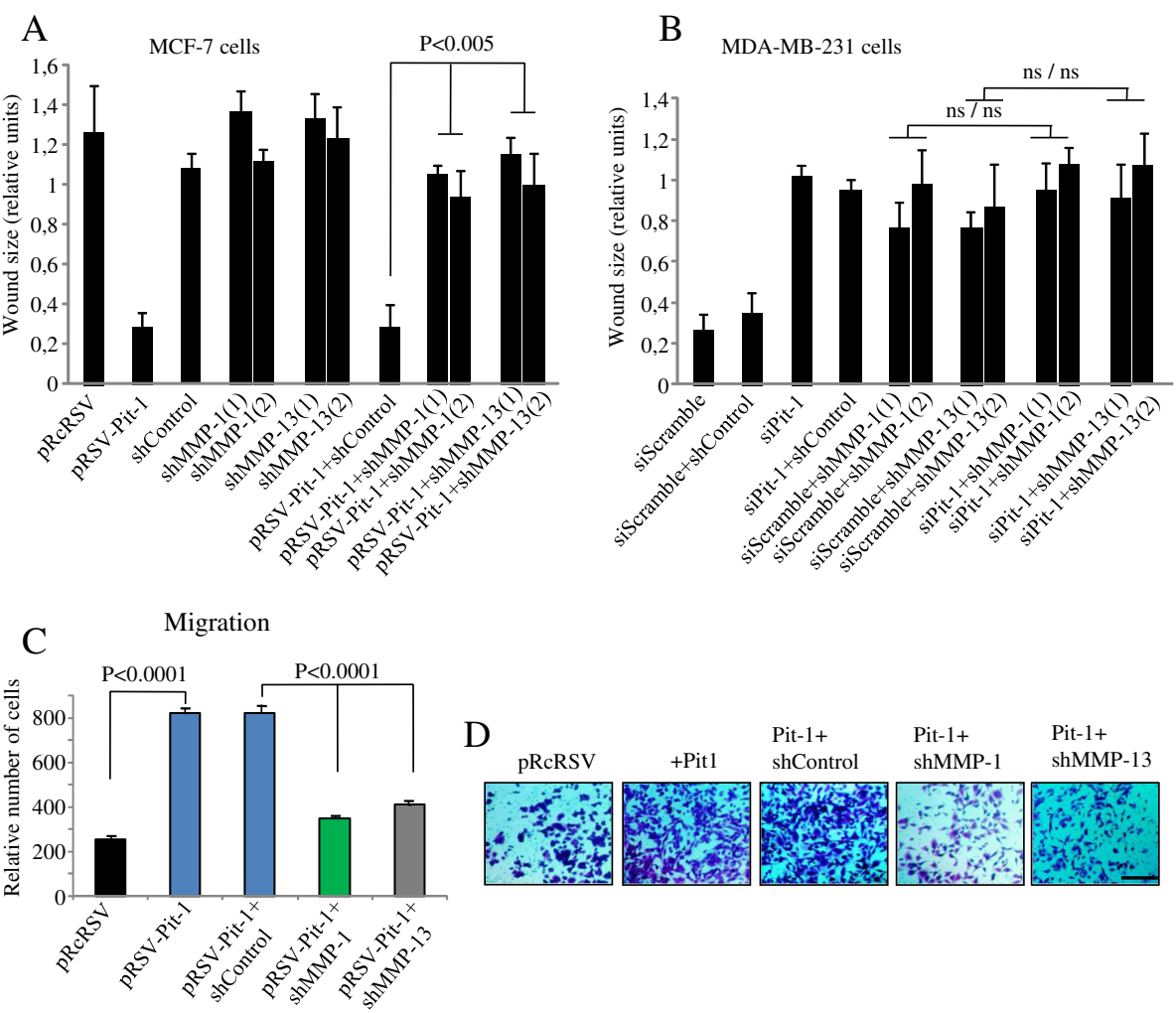

E

Invasion

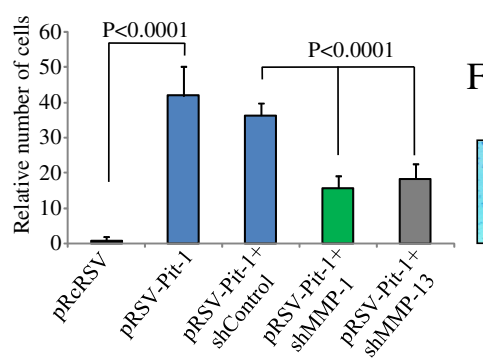

F pRcRSV +Pit1

Pit-1+
sh Control

Pit-1+

Pit-1+

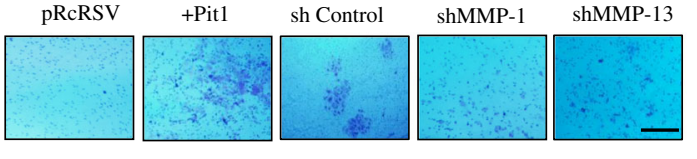

Fig. 4 MMP-1 and MMP-13 knockdown reduces invasive features in MCF-7 cells with Pit-1 overexpression, and in MDA-MB-231 cells. a-b Woundhealing assay in (a) MCF-7 cells with Pit-1 overexpression (pRSV-hPit-1), and knockdown of MMP-1 (shMMP-1(1) and shMMP-1(2)) and MMP-13 (shMMP-13(1) and shMMP-13(2)); (B) MDA-MB-231 cells with knockdown of Pit-1 (siPit-1), MMP-1 (shMMP-1(1) and shMMP-1(2)), and MMP-13 (shMMP13(1) and shMMP-13(2)). Distance between the wound edges was measured at 48 hours in three different assays, and data are represented as mean \pm SD; ns = not significant. c-d Cell motility through uncoated filters (migration) at 24 hours in control MCF-7 cells (pRcRSV), Pit-1-overexpressing MCF-7 cells (pRSV-hPit-1), and Pit-1-overexpressing and knockdown of MMP-1 or MMP-13 MCF-7 cells (pRSV-hPit-1 + shMMP-1 or -13). e-f Cell motility through matrigel-coated filters at 48 hours in control cells, cells transfected with the pRSV-hPit-1 vector, and cells transfected with pRSV-hPit-1 and knockdown of MMP-1 (Pit-1 + shMMP-1) or MMP-13 (Pit-1 + shMMP-13). Numbers represent mean \pm SD. Scale bar: 100 m. MMP-1, matrix metalloproteinase-1; MMP-13, matrix metalloproteinase-13; Pit-1, POU class 1 homeobox 1 
